\title{
CEFALÓPODES CAPTURADOS PELA PESCA COMERCIAL DE TALUDE NO SUDESTE E SUL DO BRASIL
}

\author{
PEREZ, J.A.A. ${ }^{1}$; MARTINS, R.S. ${ }^{1}$ \& R.A. SANTOS ${ }^{2}$ \\ ${ }^{1}$ Centro de Ciências Tecnológicas da Terra e do Mar, Universidade do Vale do Itajaí \\ (CTTMar-UNIVALI) e-mail: angel.perez@univali.br \\ ${ }^{2}$ Centro de Pesquisa e Extensão Pesqueira do Sudeste e Sul do Brasil \\ (CEPSUL-IBAMA) \\ *Convênio SEAP / UNIVALI - SEAP-PR/01/2003; SEAP-PR/78/2004
}

\begin{abstract}
RESUMO
O desenvolvimento da pesca demersal em áreas do talude do Sudeste e Sul do Brasil tomou impulso a partir de 2000, com operações de embarcações nacionais e arrendadas atuantes nas modalidades de espinhel-de-fundo, covos, emalhe-de-fundo e arrasto-de-fundo. Deste então essas operações vêm sendo continuamente monitoradas por Observadores de Bordo, como parte das atividades do Grupo de Estudos Pesqueiros (CTTMar-UNIVALI) no âmbito dos convênios com o Governo Federal (Ministério da Agricultura Pecuária e Abastecimento; Secretaria Especial de Aqüicultura e Pesca). Além das análises direcionadas à avaliação dos potenciais de captura dos principais recursos, esforços têm sido direcionados ao estudo do impacto das pescarias de profundidade sobre organismos não-alvo, bem como sobre o ecossistema profundo como um todo. Nesse sentido o presente trabalho relaciona a fauna de moluscos cefalópodes capturados pela pesca de arrasto e de emalhe entre 2001 e 2003. Apenas o calamar-argentino Illex argentinus figurou como espécie-alvo da pesca de arrasto, sobretudo no inverno de 2001. Outras 15 espécies, distribuídas nas ordens Spirulida (1), Sepiolida (1), Teuthida (6) e Octopodida (5), fizeram parte da fauna acompanhante dessas operações. Dessas merecem destaque, pela abundância numérica em algumas viagens, as lulas Pholidoteuthis adami, Histioteuthis corona corona e o polvo Vosseledone charrua. A pesca de emalhe, direcionada ao peixe-sapo entre 2001 e 2002 , capturou, além do calamar-argentino, dois octópodes incluindo o pelágico Argonauta argo.
\end{abstract}

Palavras-chave: cefalópodes, pesca profunda, fauna acompanhante

\section{CEPHALOPODS IN THE CATCHES OF COMMERCIAL FISHERIES ON THE SLOPE GROUNDS OFF SOUTHEASTERN AND SOUTHERN BRAZIL}

\begin{abstract}
The development of demersal fishing in slope areas off southern Brazil $\left(21^{\circ}-34^{\circ} \mathrm{S}\right)$ accelerated since the year 2000 as a result of commercial operations conducted by national and chartered bottom longliners, potters, gillnetters and trawlers. These operations were continuously monitored by observers as part of scientific research program carried out by the Fisheries Study Group (CTTMar - UNIVALI) in collaboration with Brazilian Government. Besides the assessment of the main stocks, efforts have been concentrated on assessing the impact generated by fishing on non-target organisms and on deep-water ecosystem as a whole. In this context, this work lists cephalopod fauna caught by both trawling and gillnetting between 2001 and 2003. Only the argentinean squid IIlex argentinus was included between trawlers target-species, specially during the winter of 2001. Other 15 species, included in the orders Spirulida (1), Sepiolida (1), Teuthida (6) and Octopodida (5), were caught as by-catch of trawling operations. Particularly abundant in
\end{abstract}


PEREZ et al.: Cefalópodes na pesca comercial de talude.

some fishing trips were the squid Pholidoteuthis adami and Histioteuthis corona corona, and the octopus Vosseledone charrua. Gillnet fishing directed towards the monkfish between 2001 and 2002 caught, besides the argentinean squid, two octopods including the pelagic Argonauta argo.

Keywords: Cephalopods, deepwater fishing, bycatch

\section{INTRODUÇÃO}

O desenvolvimento da pesca demersal em áreas do talude do Sudeste e Sul do Brasil tomou impulso a partir do ano 2000, com a expansão das áreas de pesca de arrasto para a borda da plataforma e o início das operações de embarcações arrendadas atuantes nas modalidades de espinhel-de-fundo, covos, emalhe-de-fundo e arrasto-de-fundo (Perez et al., 2001; Perez et al., 2002; Perez et al., 2003; Pezzuto et al., 2002; Pezzuto et al., 2004a e b). Esse processo promoveu a rápida estruturação de pescarias profundas direcionadas à espécies de peixes, como o peixe-sapo (Lophius gastrophysus), a merluza (Merluccius hubbsi), a abrótea-de-profundidade (Urophycis cirrata), o galo-de-profundidade (Zenopsis conchiffer) e arraias emplastro (Atlantoraja spp.), bem como alguns invertebrados como os caranguejos real (Chaceon ramosae) e vermelho (C. notialis) e os camarões de profundidade (Fam. Aristeidae). O calamar-argentino Illex argentinus foi o único molusco cefalópode a figurar entre as espécies-alvo dessas pescarias, sustentando sazonalmente importantes capturas da pesca de arrasto (Silva et al., 2002; Silva, 2003). Essa modalidade de pesca capturou numerosos invertebrados bentônicos (Bastos, 2004; Bastos et al., 2004) e inclusive outros cefalópodes, que não foram aproveitados comercialmente e compuseram o rejeito dessas operações.

O desempenho e o potencial de sustentabilidade das pescarias profundas no Sudeste e Sul do Brasil têm sido avaliados a partir do monitoramento contínuo das operações da pesca arrendada por Observadores de Bordo, como parte das atividades do Grupo de
Estudos Pesqueiros (CTTMar-UNIVALI) no âmbito dos convênios com o Governo Federal (Ministério da Agricultura Pecuária e Abastecimento; Secretaria Especial de Aqüicultura e Pesca). Além das análises direcionadas à avaliação dos potenciais de captura dos principais recursos, esforços têm sido direcionados ao estudo do impacto das pescarias de profundidade sobre organismos não-alvo, bem como sobre o ecossistema profundo como um todo. Uma análise detalhada da pesca de emalhe direcionada ao peixe-sapo entre 2001 e 2002 , dimensionou o impacto da mortalidade não intencional gerada pela frota arrendada sobre cerca de 100 espécies incluindo-se peixes, invertebrados, mamíferos, aves e répteis (Perez $\&$ Wahrlich, 2004). Além disso, cerca 170 espécies de invertebrados, componentes da fauna acompanhante gerada pelas operações de pesca arrendada de arrasto, emalhe e covos, foram identificados e descritos para uma avaliação qualitativa do impacto dessas pescarias sobre os habitats bentônicos no Sudeste e Sul do Brasil (Bastos, 2004; Bastos et al., 2004). Essa análise abrangeu espécies de poríferos, cnidários, poliquetos, crustáceos, equinodermos e moluscos gastrópodes e bivalves. O presente trabalho relaciona os moluscos cefalópodes capturados pela pesca de arrasto e de emalhe entre 2001 e 2003, em complementação a essa primeira revisão qualitativa da fauna de invertebrados acompanhantes da pesca profunda.

\section{MATERIAL E MÉTODOS}

O material examinado foi coletado durante 19 viagens de pesca realizadas por sete embarcações arrendadas e duas nacionais nas 
áreas de talude do Sudeste e Sul do Brasil entre 2001 e 2003 (Tabela 1). Com exceção de duas viagens realizadas por embarcações arrendadas de pesca de emalhe direcionada ao peixesapo (Lophius gastrophysus), todas as demais viagens corresponderam à operações de pesca de arrasto de fundo.

Todas as viagens da frota arrendada foram monitoradas por Observadores de Bordo, como parte do Programa de Observadores de Bordo executado desde 2000 no âmbito dos convênios celebrados entre a Universidade do Vale do Itajaí e o Ministério da Agricultura, Pecuária e Abastecimento (MAPA/ SARC/ 03/ 2000; MAPA/ SARC/ DPA/ 03/ 2001; MAPA/ SARC/ DENACOOP/ 176/ 2002) e a Secretaria Especial de Aqüicultura e Pesca (SEAP/ PR/ 01/2003; SEAP/PR/ 078/2004). Após cada lance, os Observadores amostraram a composição total da captura incluindo as espéciesalvo e a fauna acompanhante. Os organismos da fauna acompanhante foram separados da captura total, fotografados e, principalmente no caso dos cefalópodes, embalados e congelados para posterior identificação em laboratório. Detalhes sobre esses procedimentos são apresentados em Perez \& Wahrlich (2004) e Bastos (2004).

O material proveniente das duas viagens realizadas por arrasteiros nacionais foi congelado a bordo e fornecido pela própria tripulação das embarcações aos coletores do Programa de Estatística Pesqueira do CTTMar-UNIVALI após o desembarque no porto de Navegantes, Santa Catarina.

Em laboratório o material foi descongelado e procedeu-se a identificação a partir das revisões sistemáticas recentes (Nesis, 1987; Voss, et al., 1998). Os espécimes foram pesados e tiveram os sexos determinados. A morfometria de algumas espécies foi realizada de acordo com as medidas definidas em Roper \& Voss (1983).

\section{RESULTADOS E DISCUSSÃO}

O calamar-argentino Illex argentinus (Castellanos, 1960) figurou como espécie-alvo da pesca de arrasto, sobretudo no inverno de 2002 com capturas em torno de 230 t. A espécie foi registrada como um componente pouco expressivo do fauna acompanhante da pesca de emalhe direcionada ao peixe-sapo (Perez \& Wahrlich, 2004) e foi objeto de estudos de biologia e pesca a partir das volumosas capturas geradas pela pesca de arrasto (Perez et al., 2003; Silva et al., 2002; Silva, 2003). Por essa razão, não foi enfocada no presente trabalho.

A maioria dos 217 exemplares examinados proveio das capturas de arrasteiros operantes nos fundos de talude da costa dos estados de São Paulo e Rio de Janeiro, principalmente direcionados à captura da merluza (Merluccius hubbsi), entre 300 e 400 m de profundidade, e aos camarões-de-profundidade (Família Aristeidae) entre 600 e 800 m (Figura 1). Foram identificadas 15 espécies, distribuídas nas ordens Spirulida (1), Sepiolida (1), Teuthida (6) e Octopodida (5) (Tabela 2), entre as quais merecem destaque, pela elevada freqüência em algumas viagens, as lulas Pholidoteuthis adami Voss, 1956, Histioteuthis corona corona (Voss \& Voss, 1962) e o polvo Vosseledone charrua Palácio, 1978. A pesca de emalhe, direcionada ao peixe-sapo entre 2001 e 2002, capturou, além do calamar-argentino, dois octópodes incluindo o pelágico Argonauta argo (Linnaeus, 1758).

Pholidoteuthis adami é uma lula de grande tamanho pertencente à família Lepidoteuthidae, cuja característica principal é o manto recoberto por tubérculos. A espécie ocorre apenas no lado oeste do Atlântico desde a Nova Inglaterra até o Uruguai (Nesis, 1987), sendo associada aos fundos batiais entre 500 e $2000 \mathrm{~m}$ de profundidade. Um total de 40 indivíduos capturados por arrastos de fundo foram examinados sendo 19 machos, 18 fêmeas e 3 indivíduos com sexo indeterminado (Tabela 3). 
PEREZ et al.: Cefalópodes na pesca comercial de talude.

Tabela 1 - Sumário das viagens de pesca profunda realizadas entre 2000 e 2003 no Sudeste e Sul do Brasil, que originaram os cefalópodes em sua fauna acompanhante. a, arrendado; $n$, nacional

\begin{tabular}{|c|c|c|c|c|c|}
\hline Embarcação & Data de saída & $\begin{array}{c}\text { Data de } \\
\text { chegada }\end{array}$ & $\begin{array}{c}\text { Latitude } \\
\text { (mín-máx) }\end{array}$ & $\begin{array}{l}\text { Longitude } \\
\text { (mín-máx) }\end{array}$ & $\begin{array}{l}\text { Profundidade } \\
\text { (mín-máx) }\end{array}$ \\
\hline \multirow[t]{2}{*}{$\mathrm{Cipi}^{\mathrm{a}}$} & 04/10/01 & $19 / 11 / 01$ & $\begin{array}{l}-23,391 \\
-33,076\end{array}$ & $\begin{array}{l}-41,753 \\
-50,456\end{array}$ & $224-510$ \\
\hline & 29/11/01 & 02/01/02 & $\begin{array}{l}-23,682 \\
-26,478\end{array}$ & $\begin{array}{l}-41,848 \\
-46,512\end{array}$ & $245-439$ \\
\hline \multirow[t]{5}{*}{ Costa Grande $^{a}$} & 03/04/02 & $12 / 05 / 02$ & $\begin{array}{l}-23,667 \\
-24,840\end{array}$ & $\begin{array}{l}-41,770 \\
-44,633\end{array}$ & $220-750$ \\
\hline & $15 / 05 / 02$ & 26/06/02 & $\begin{array}{l}-23,658 \\
-25,279\end{array}$ & $\begin{array}{l}-41,823 \\
-44,931\end{array}$ & $265-762$ \\
\hline & $17 / 08 / 02$ & 24/09/02 & $\begin{array}{l}-23,083 \\
-24,913\end{array}$ & $\begin{array}{l}-41,968 \\
-44,567\end{array}$ & $310-435$ \\
\hline & $13 / 01 / 03$ & 05/03/03 & $\begin{array}{l}-23,851 \\
-24,073 \\
\end{array}$ & $\begin{array}{l}-41,135 \\
-42,960 \\
\end{array}$ & $722-777$ \\
\hline & $10 / 03 / 03$ & $23 / 05 / 03$ & $\begin{array}{l}-23.247 \\
-24,412 \\
\end{array}$ & $\begin{array}{l}-41,195 \\
-44,240 \\
\end{array}$ & $247-750$ \\
\hline \multirow[t]{3}{*}{ Insung $207^{a}$} & $20 / 10 / 01$ & 01/12/01 & $\begin{array}{l}-23,146 \\
-27,885\end{array}$ & $\begin{array}{l}-42,161 \\
-48,644\end{array}$ & $196-396$ \\
\hline & 28/05/02 & $14 / 07 / 02$ & $\begin{array}{l}-22,833 \\
-33,322\end{array}$ & $\begin{array}{l}-40,723 \\
-50,518\end{array}$ & $180-700$ \\
\hline & 02/10/02 & $28 / 10 / 02$ & $\begin{array}{l}-23,807 \\
-29,201 \\
\end{array}$ & $\begin{array}{l}-42,740 \\
-47,941 \\
\end{array}$ & $212-536$ \\
\hline \multirow[t]{4}{*}{ Nuevo Apenino ${ }^{a}$} & $17 / 09 / 01$ & $16 / 10 / 01$ & $\begin{array}{l}-23,660 \\
-24,292\end{array}$ & $\begin{array}{l}-41,950 \\
-48,830\end{array}$ & $186-548$ \\
\hline & $24 / 10 / 01$ & $12 / 11 / 01$ & $\begin{array}{l}-23,000 \\
-24,923\end{array}$ & $\begin{array}{l}-41,017 \\
-44,683\end{array}$ & $305-448$ \\
\hline & $24 / 02 / 02$ & $30 / 03 / 02$ & $\begin{array}{l}-23,680 \\
-26,794\end{array}$ & $\begin{array}{l}-41,870 \\
-46,101\end{array}$ & $313-797$ \\
\hline & 05/04/02 & $28 / 04 / 02$ & $\begin{array}{l}-23,633 \\
-24,863\end{array}$ & $\begin{array}{l}-41,625 \\
-48,312\end{array}$ & $329-768$ \\
\hline Rio Bouzos Uno ${ }^{a}$ & $11 / 05 / 02$ & $27 / 06 / 02$ & $\begin{array}{l}-23,682 \\
-27,148\end{array}$ & $\begin{array}{l}-41,924 \\
-46,846\end{array}$ & $288-406$ \\
\hline Leardini $^{n}$ & várias viagens & em 2002 & & $-S$ & $>200 \mathrm{~m}$ \\
\hline Capitão Lucas $^{n}$ & $06 / 10 / 02$ & $18 / 10 / 02$ & \multicolumn{3}{|c|}{ SE Cabo de Sta Marta Grande } \\
\hline Juno $^{a}$ & 21/03/01 & $13 / 05 / 01$ & $\begin{array}{l}-28,816 \\
-31,013\end{array}$ & $\begin{array}{l}-47,636 \\
-49,323\end{array}$ & $250-495$ \\
\hline Belen $^{a}$ & $17 / 06 / 02$ & $20 / 08 / 02$ & $\begin{array}{l}-24,306 \\
-25,068\end{array}$ & $\begin{array}{l}-43,781 \\
-44,970\end{array}$ & $215-460$ \\
\hline
\end{tabular}

Todos os exemplares apresentaram coloração avermelhada sobre as faces ventral e dorsal do manto, com nadadeiras, cabeça e braços despigmentados (Figura 2). Os machos capturados variaram entre 405 e $2250 \mathrm{~g}$ e as fêmeas entre 1445 e 8510 g. A maior fêmea mediu 778 mm de $\mathrm{CM}$, valor próximo do máximo observado para a espécie $(780 \mathrm{~mm})$ (Roper et al., 1984). Os comprimentos das nadadeiras foram no máximo $68 \%$ do $\mathrm{CM}$, valor inferior ao descrito para a espécie (70-75\%). O braço mais longo atingiu $83 \%$ do $\mathrm{CM}$ (Tabela 3 ). A maior 
parte dos exemplares foram provenientes das operações de pesca dirigidas aos camarõesde-profundidade (Fam. Aristeidae) entre $23^{\circ} \mathrm{e}$ $24^{\circ} \mathrm{S}$ e 710 e $770 \mathrm{~m}$ de profundidade (Figura 1).

Incluída na família Histioteutidae, que tem apenas um gênero, Histioteuthis corona corona são lulas medianas a grandes, com um manto curto e o corpo recoberto por fotóforos (Nesis, 1987; Voss et al., 1998). A espécie foi capturada com freqüência por arrasteiros sobre fundos entre 716 e $777 \mathrm{~m}$ de profundidade. Foram examinados 24 machos, 15 fêmeas e 4 indivíduos com sexo indeterminado. O maior macho e a maior fêmea mediram 234 e $251 \mathrm{~mm}$
$\mathrm{CM}$ respectivamente. A espécie é cosmopolita e politípica, sendo que outras quatro sub-espécies habitam águas tropicais e subtropicais do oceano Pacífico. Sua distribuição no Atlântico está fortemente relacionada às águas do Caribe, Golfo do México e as áreas subtropicais do Atlântico Norte, sob influência da Corrente do Golfo. Sua presença no Atlântico sul foi documentada para águas tropicais do lado oriental, até o Golfo da Guiné. Apenas o registro de uma paralarva é conhecido para o setor oeste, sugerindo que a distribuição da espécie fosse confinada apenas às áreas mais produtivas do Atlântico sul (Voss et al., 1998). Os registros gerados pela pesca comercial na costa do Bra-

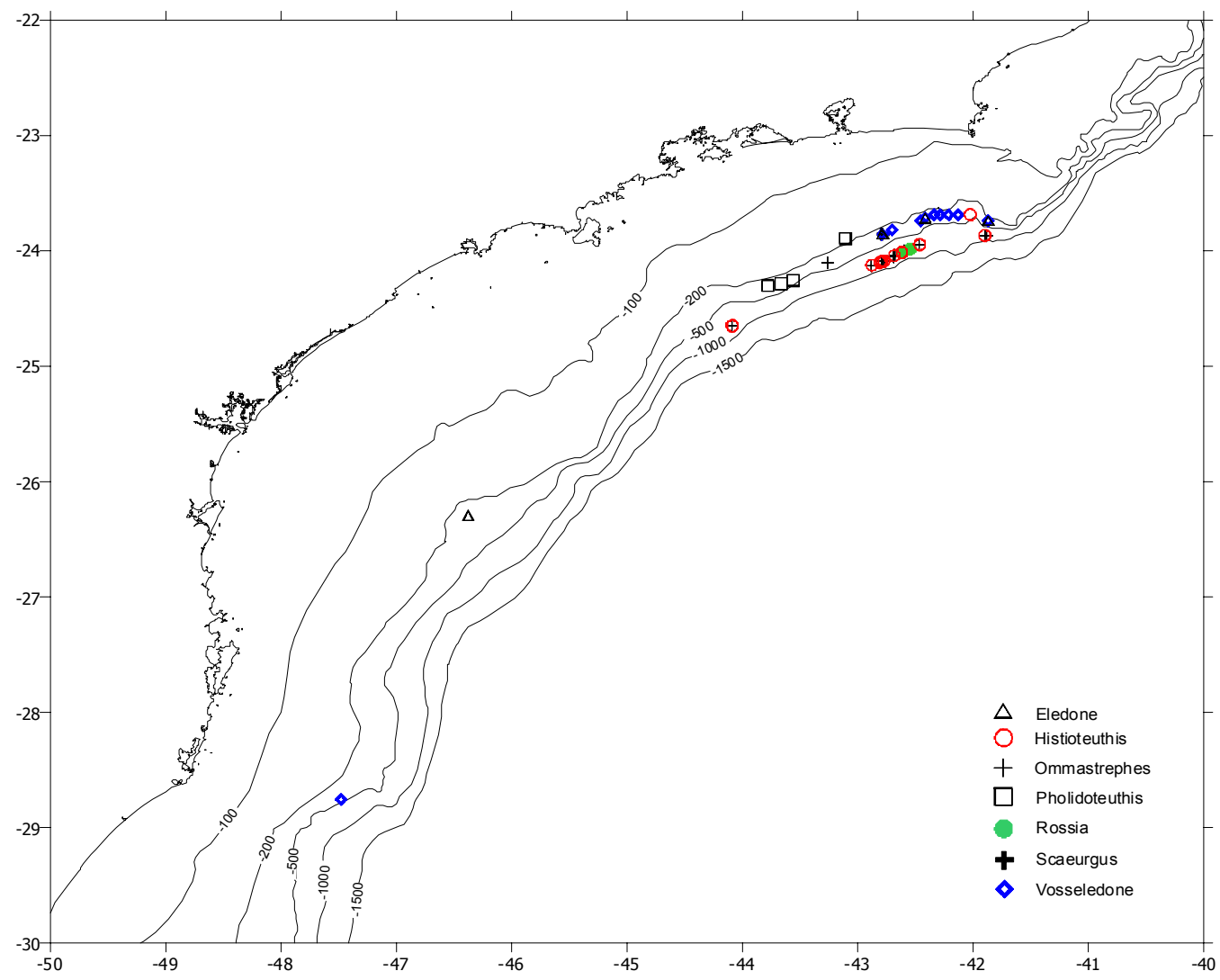

Figura 1 - Distribuição espacial dos lances de pesca de arrasto de fundo com captura de cefalópodes como fauna acompanhante. 
PEREZ et al.: Cefalópodes na pesca comercial de talude.

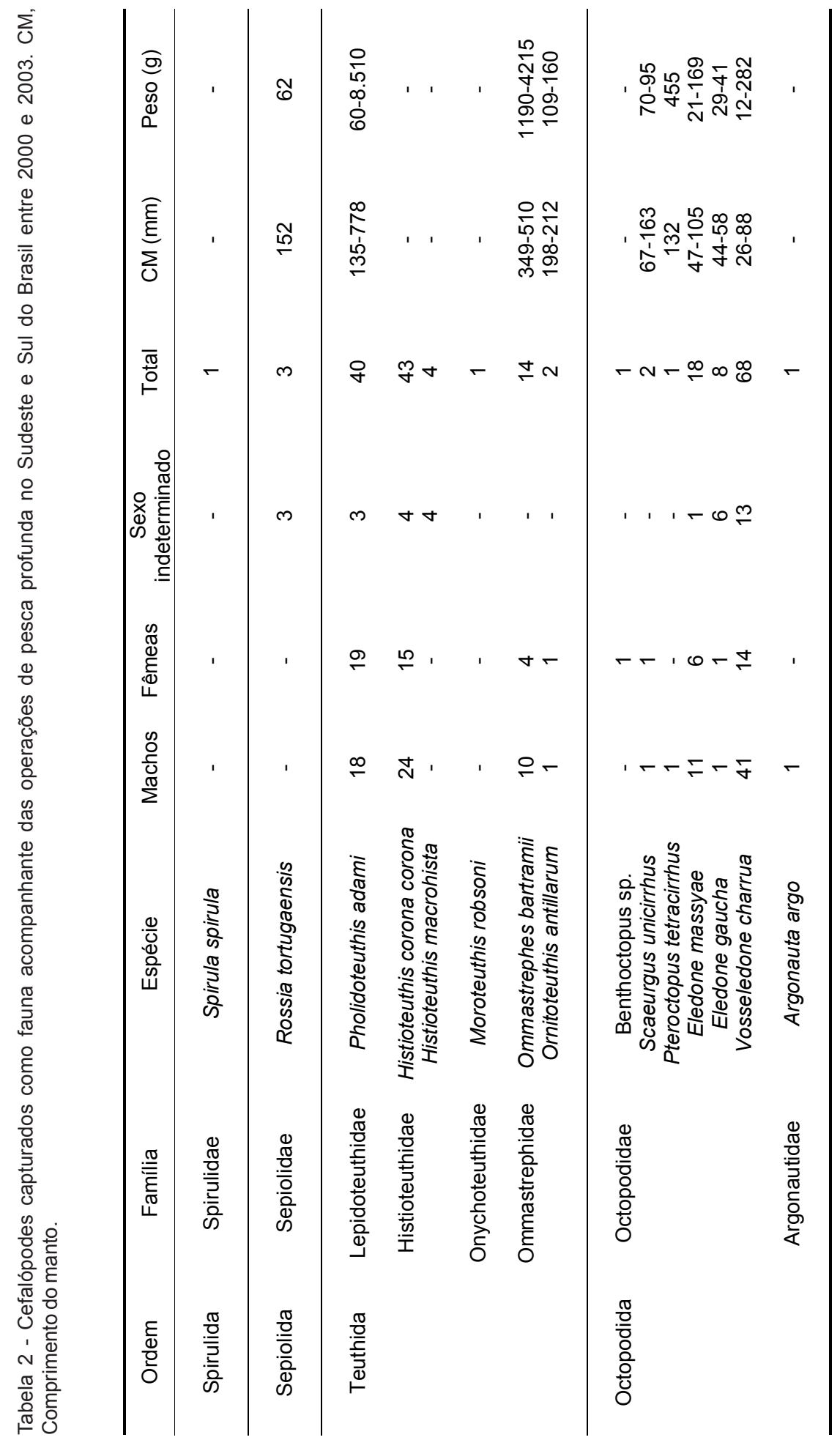


Tabela 3 - Morfometria de 18 machos e 19 fêmeas de Pholidoteuthis adami capturado como fauna acompanhante da pesca de arrasto profundo no Sudeste e Sul do Brasil entre 2000 e 2003.

\begin{tabular}{ccccc}
\hline & \multicolumn{2}{c}{ Machos } & \multicolumn{2}{c}{ Fêmeas } \\
& Máximo & Mínimo & Máximo & Mínimo \\
\hline Peso & 2250 & 405 & 8510 & 1445 \\
Comprimento do manto CM & 544 & 345 & 778 & 458 \\
Largura do manto LM & 160 & 76 & 270 & 117 \\
Comprimento da cabeça CC & 86 & 55 & 115 & 65 \\
Largura da cabeça LC & 60 & 34 & 84 & 43 \\
Comprimento da nadadeira CN & 363 & 202 & 540 & 275 \\
Largura da nadadeira LN & 175 & 86 & 250 & 124 \\
Comprimento do funil Cfu & 60 & 34 & 109 & 43 \\
Largura da base do funil Lfu & 49 & 25 & 72 & 37 \\
Diâmetro do olho DO & 49 & 28 & 47 & 29 \\
Comprimento do braço direito I CBI & 340 & 206 & 363 & 236 \\
Comprimento do braço direito II CBII & 370 & 196 & 382 & 310 \\
Comprimento do braço direito III CBIII & 341 & 247 & 358 & 245 \\
Comprimento do braço direito IV CBIV & 323 & 270 & 343 & 273 \\
Comprimento do tentáculo CT & 1437 & 857 & 1423 & 857 \\
\hline & & & & \\
LM/CM & 0,21 & 0,10 & 0,35 & 0,15 \\
CC/CM & 0,18 & 0,11 & 0,21 & 0,11 \\
LC/CC & 0,81 & 0,56 & 1,01 & 0,59 \\
CN/CM & 0,67 & 0,48 & 0,69 & 0,48 \\
CN/LN & 0,60 & 0,39 & 0,76 & 0,41 \\
Cfu/CM & 0,12 & 0,07 & 0,14 & 0,08 \\
LFu/CM & 1,09 & 0,54 & 0,92 & 0,64 \\
DO/CM & 0,11 & 0,05 & 0,09 & 0,05 \\
CBI/CM & 0,63 & 0,39 & 0,73 & 0,42 \\
CBII/CM & 0,70 & 0,57 & 0,83 & 0,40 \\
CBIII/CM & 0,69 & 0,59 & 0,76 & 0,42 \\
CBIV/CM & 0,66 & 0,50 & 0,73 & 0,37 \\
CT/CM & 3,06 & 2,22 & 2,69 & 1,39 \\
\hline \hline
\end{tabular}

sil, em torno dos $24^{\circ} \mathrm{S}$ e entre $42-43^{\circ} \mathrm{W}$, revelam a presença da espécie também na zona tropical do Atlântico Sudoeste.

Um único exemplar de Moroteuthis robsoni Adam,1962 foi obtido através da captura da pesca de arrasto. A espécie pertence à família Onychoteuthidae que se caracteriza pela presença de robustos ganchos quitinosos nas massas tentaculares (Figura 3). M. robsoni tem nadadeiras com terminação em ponta, pele rugosa coberta por tubérculos e se distribui no mundo todo desde áreas subtropicais até o Oceano Antártico (Nesis, 1987).

Os octópodes bentônicos Eledone massyae Voss, 1965 e E. gaucha, Haimovici, 1988 , são relativamente abundantes no Sudeste e Sul do Brasil, tendo sido registrados entre 60 e $300 \mathrm{~m}$ de profundidade (Haimovici \& Perez, 1991; Perez \& Haimovici, 1995). As capturas da pesca arrasto de talude confirmou a pre- 

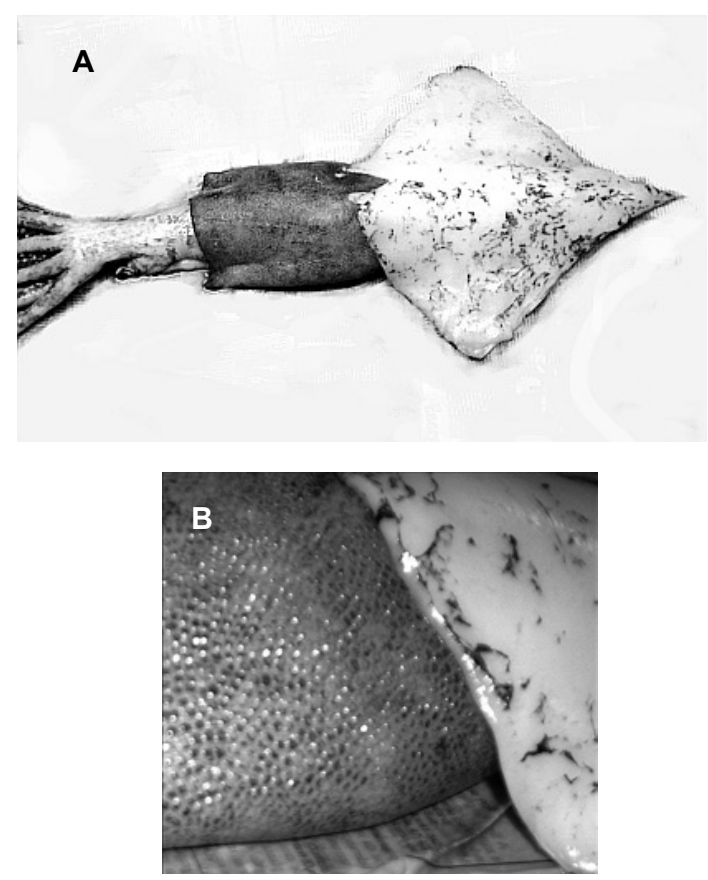

Figura 2 - Pholidoteuthis adami capturado como fauna acompanhante da pesca de arrasto profundo no Sudeste do Brasil, O exemplar fêmea tinha $45 \mathrm{~cm}$ de comprimento do manto. $A$, exemplar inteiro; $B$, detalhe as superfície dorsal do manto.

sença das duas espécies nas áreas mais ao norte da distribuição conhecida (litoral de São Paulo e Rio de Janeiro) e profundidades entre 300 e 400 m. Onze machos, seis fêmeas e um exemplar de sexo indeterminado de E. massyae foram examinados, sendo notada a presença de machos grandes (Tabela 2).

Vosseledone charrua foi o cefalópode não comercial mais freqüentemente capturado pela pesca comercial de arrasto no talude do Sudeste e Sul do Brasil (Tabela 1). A espécie tem coloração avermelhada, pele rugosa e machos apresentam hectocotilo diferenciado com lígula em forma de colher e calimus conspícuo (Figura 3). A presença da espécie ao norte da costa do Rio Grande do Sul era suportada por um único registro em Palácio (1978) considerado duvidoso (Haimovici \& Perez, 1991). As numerosas capturas obtidas entre 300 e 400
A

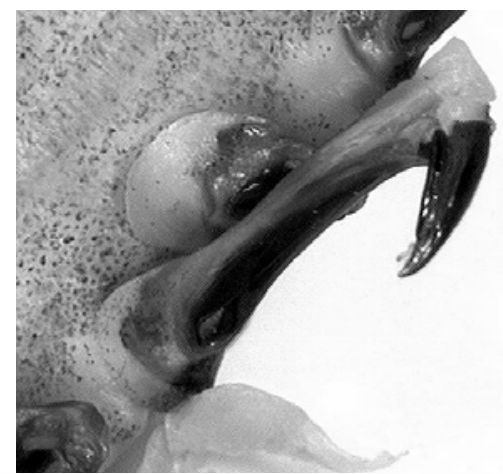

B

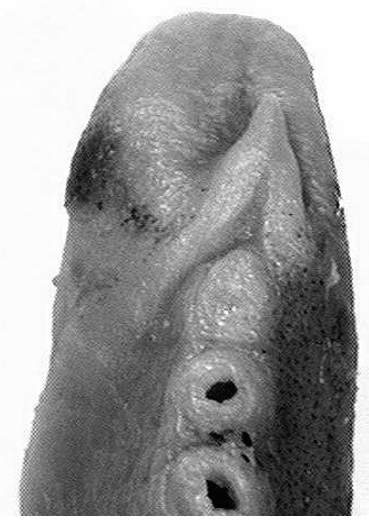

Figura 3 - Detalhes da morfologia externa de dois cefalópoes capturados como fauna acompanhante da pesca de arrasto profundo no Sudeste e Sul do Brasil. (A) Moroteuthis robsoni, detalhe dos ganchos presentes nas massas tentaculares; (B) Vosseledone charrua, detalhe da extremidade do hectocotilo.

$\mathrm{m}$ confirmam que a espécie efetivamente estende sua distribuição até o litoral de São Paulo e Rio de Janeiro. Foram examinados 41 machos, 14 fêmeas e 13 indivíduos de sexo indeterminado sendo ambos os sexos similares em comprimento do manto e peso (machos 39-88 mm CM - 23-282 g; fêmeas 43-80 mm $\mathrm{CM}-28-143 \mathrm{~g}$ ).

Os polvos Scaeurgus unicirrhus (Delle Chiage, 1830), Pteroctopus tetracirrhus (Delle Chiage, 1830) e Benthoctopus sp., também foram registrados nas operações de pesca de arrasto. Os dois primeiros são típicos de águas tropicais e subtropicais e ocorrem desde águas sublitorais até $400-700 \mathrm{~m}$ de profundidade (Nesis, 1987). O gênero Benthoctopus inclue 
entre 15 e 23 espécies distribuídas em todos os oceanos. Nas latitudes tropicais o grupo se apresenta em profundidades maiores que 500 m (Nesis, 1987).

O presente trabalho dá continuidade à análise do impacto da atividade pesqueira comercial em algumas regiões do talude das regiões Sudeste e Sul do Brasil (Perez \& Wahrlich, 2004; Bastos, 2004). Várias espécies registradas neste levantamento como Rossia tortugaensis, $P$. adamis e $M$. robsoni não estão incluídas nas revisões mais recentes da fauna de cefalópodes da costa do Brasil. No entanto, estas revisões concentraram-se principalmente nas áreas costeiras e de plataforma continental (Haimovici \& Perez, 1991; Perez \& Haimovici, 1991; Haimovici et al., 1994). Esforços recentes para explorar o potencial pesqueiro de áreas de talude têm compilado inúmeros registros novos de espécies de águas profundas o que deve permitir, no futuro, uma revisão mais completa da fauna de cefalópodes do Brasil.

\section{REFERÊNCIAS BIBLIOGRÁFICAS}

Bastos, M. 2004. Invertebrados bentônicos capturados incidentalmente pela frota pesqueira arrendada no sudeste e sul do Brasil. Trabalho de Conclusão de Curso de Oceanografia. Centro de Ciências Tecnológicas da Terra e do Mar. Universidade do Vale do Itajaí.

Bastos, M.; Pezzuto, P.R. \& R. Wahrlich. 2004. Invertebrados bentônicos capturados pela frota arrendada no talude do Sudeste e Sul do Brasil. 1. Padrões gerais de ocorrência dos grupos. p. 126. Livro de Resumos. Congresso Brasileiro de Oceanografia' 2004 - XVI Semana Nacional de Oceanografia, 10 a 15 de outubro de 2004. Associação Brasileira de Oceanografia - Universidade do Vale do Itajaí, Itajaí, Santa Catarina.

Haimovici, M. \& J.A.A. Perez. 1991. Coastal cephalopod fauna of southern Brazil. Bull. Mar. Sci. 49(1-2): 221-230..
Haimovici, M.; Perez, J.A.A. \& R.A. Santos. 1994. Class Cephalopoda, p. 311-320. In: Rios,E.C. (ed.) Seashells of Brazil. 2a. Edição, Editora da Furg, Rio Grande.

Nesis, K. 1987. Cephalopods of the world. Squids, cuttlefishes, and allies. T.F.H. Publications Inc. Ltd. 351p.

Perez, J.A.A. \& M. Haimovici. 1991. Cephalopod collection of "Museu de Zoologia da Universidade de São Paulo", São Paulo, Brazil. Papéis Avulsos Zool., 37(16):251-258.

Perez, J.A.A. \& M. Haimovici. 1995. Descriptive ecology of two south american eledonids (Cephalopoda: Octopodidae). Bull. Mar. Sci., 56(3): 752-766.

Perez, J.A.A. \& R. Wahrlich. 2004. A bycatch assessment of the gillnet monkfish Lophius gastrophysus fishery off southern Brazil. Fisheries Research. 72:81-95.

Perez, J. A. A.; Pezzuto, P. R.; Rodrigues, L. F.; Valentini, H. \& C.M. Vooren. 2001. Relatório da reunião técnica de ordenamento da pesca de arrasto nas regiões Sudeste e Sul do Brasil. In: Pezzuto, P. R.; Perez, J. A. A.; Rodrigues, L. F. \& Valentini, H. (eds.). Reuniões de ordenamento da pesca demersal nas regiões Sudeste e Sul do Brasil: 2000-2001. Notas Téc. FACIMAR, 5: 1-34.

Perez, J. A. A.; Wahrlich, R.; Pezzuto, P. R. \& F.R.A. Lopes. 2002. Estrutura e dinâmica da pescaria do peixe-sapo Lophius gastrophysus no Sudeste e Sul do Brasil. Boletim do Instituto de Pesca, São Paulo, 28(2):205-231.

Perez, J. A. A.; Wahrlich, R.; Pezzuto, P. R.; Schwingel, P. R.; Lopes, F. R. A. \& M. Rodrigues-Ribeiro. 2003. Deep-sea fishery off southern Brazil: recent trends of the Brazilian fishing industry. J. Northw. Atl. Fish. Sci. 31:1-18

Pezzuto, P.R.; Perez, J.A.A.; Wahrlich, R. \& W.G. Vale. 2002. Avaliação da pescaria dos caranguejos-de-profundidade no Sul do Brasil. Anos 2001-2002 Convênio UNIVALI/ MAPA (MAPA/ SARC/ DPA 03/ 2001; 
MAPA/ SARC/ DENACOOP/ 176/ 2002). Relatório Final. Itajaí, dezembro de 2002.

Pezzuto, P.R.; Perez, J.A.A. \& R. Wahrlich. 2004a. The recent expansion of the Brazilian deep-water crab (Chaceon spp.) fishery. Program and Abstracts. 3rd. Brazilian Crustacean Congress \& The Crustacean Society Meeting. Hotel Costão do Santinho, Florianópolis, Santa Catarina State, Brazil. October 24 to 28, 2004.

Pezzuto, P.R.; Perez, J.A.A. \& R. Wahrlich. 2004b. Deep-sea shrimps (Decapoda: Aisteidae): new targets of the deep-water trawling fishery off the southeastern Brazilian coast. Program and Abstracts. 3rd. Brazilian Crustacean Congress \& The Crustacean Society Meeting. Hotel Costão do Santinho, Florianópolis, Santa Catarina State, Brazil. October 24 to 28, 2004.

Roper, C.F. \& G.L. Voss. 1983. Guidelines for taxonomic descriptions of cephalopod species. Memoirs of the National Museum Victoria. No. 44, p.49-63.

Roper, C.F.E.; Sweeney, M. \& C.E. Nauen. 1984. FAO species catalogue, Vol. 3. Cephalopods of the World. FAO Fisheries Synopsis No. 125, 3:227p.
Silva, T.N. 2003. Estrutura populacional e biologia reprodutiva do calamar argentino, Illex argentinus (Castellanos, 1960) (Cephalopoda: Ommastrephidae), capturado pela frota comercial de arrasto no Sudeste-Sul do Brasil. 2003. Trabalho de Conclusão de Curso (Graduação em Oceanografia) - Universidade do Vale do Itajaí.

Silva, T.N.; Perez, J.A.A. \& R.S. Martins. 2002. Estudo preliminar da reprodução e estrutura populacional do calamar-argentino, Illex argentinus (Castellanos, 1960), capturado pela frota de arrasto brasileira e arrendada no Sudeste-Sul do Brasil. Programa e Índice de Trabalhos. Simpósio Brasileiro de Oceanografia. Universidade de São Paulo, Instituto Oceanográfico. 26 a 30 de agosto de 2002, São Paulo, SP, Brasil.

Voss, N.A.; Nesis, K. \& P.G. Rodhouse. 1998. The cephalopod family Histioteuthidae (Oegopsida): systematics, biology, and biogeography. In. Noss, N.A., Vecchione, M.; Toll, R.B. \& M.J. Sweeney (eds.) Systematics and Biogeography of Cephalopods Vol. II. Smithsonian Contributions to Zoology, 586, p. 293-372. 